\title{
The Ratio Table: a tool for understanding ratios
}

\author{
Philip Southey \\ Department of Physics, University of Stellenbosch, South Africa, 7600
}

Arnold Arons described an inadequate understanding of ratios as "one of the most serious impediments to the study of science" [1]. Yet ample math and physics education research demonstrates that STEM university students struggle with ratios. For example, students are familiar with the notion of "meters per second", but many do not attribute meaning to the notion of "seconds per meter" [2]. This manuscript presents a simple technique dubbed "The Ratio Table" for helping students work algebraically with ratios, and make physical sense of units involving "per". The technique was implemented and assessed through repeated pre- and post administration of a set of written questions. Results suggest that this relatively small intervention can boost student performance. 


\section{INTRODUCTION}

\section{A. Background and Motivation}

There is a significant amount of research on ratios and proportional reasoning, particularly in mathematics education research. (See Tourniaire \& Pulos [3] and Thompson \& Saldana [4] for a review). All previous research emphasizes the importance of this skill for success in STEM subjects, yet there appears to be no formalized, generally accepted, cohesive method for understanding and applying proportional reasoning at the tertiary level.

Proportional reasoning is ubiquitous in first year undergraduate STEM subjects such as physics and chemistry. Students are expected to work with ratios when solving problems, but are often not formally taught a technique for doing so at the tertiary level. A consistent observation in the physics education literature is that students often experience difficulty with mathematically representing (mathematizing) physics problems, rather than with the physics concepts themselves [5]. In the context of proportional reasoning "mathematization" includes the ability to map a relationship between two physical quantities into 'mathematical space' [6]. The Ratio Table is a tool which directly support this process of mathematization. The Ratio Table is nothing new; it is simply the formalization of a well-known technique for working with ratios. It emphasizes the relationship between two physical quantities, mapping these quantities to two separate columns of a table. This allows a clear visuospatial representation of this mathematical relationship [7]. It is also helpful in making meaning of units involving "per”, such as kilograms per cubic meter, $\mathrm{kg} \mathrm{m}^{-3}$. (This is explained fully in section II c below). In formalizing the notion of the Ratio Table, one can more easily identify this type of technique in different contexts across different disciplines, and contrast it with other techniques such as the unit factor method (also known as dimensional analysis).

'Ratio and proportion' is a very broad topic, and highly context dependent. For example, in a 3000 student study, Boudreaux, Kanim, \& Brahmia [2] delineated six different modes of reasoning about ratios, and investigated the effect of context on students' reasoning. While the current study is concerned with three of these six modes of reasoning, a different categorization scheme is introduced for reasons outlined below.

\section{B. Numeric versus algebraic: a pilot study}

A pilot study (conducted by Southey, Chellan, Cloete, Grobbelaar and van Wyk) revealed a marked difference in how students cope with ratios involving numerical values (hereafter known as 'numeric ratios') and ratios involving algebraic values (hereafter known as ‘algebraic ratios'). The 101 freshman engineering students in the pilot obtained an average of $93 \%$ for the two questions involving numeric ratios and an average of $54 \%$ for the 4 questions involving algebraic ratios. These were therefore adopted as primary categories in the main study. Student sensitivity to "numeric" versus "algebraic” is well illustrated in a study by Torigoe and Gladding [8]. I also designated a third category, 'units involving "per”, , as students also seem to struggle to understand these types of units in terms of a ratio. For example, students are familiar with the notion of "meters per second", but many do not attribute meaning to the notion of "seconds per meter"; see the results from question 4 below.

\section{METHODOLOGY}

\section{A. Sample}

The sample of students comprised 311 engineering freshmen at the University of Stellenbosch, a dual medium university in South Africa. 115 of these students were in an English-medium class, and 203 in an Afrikaans-medium class. The pre- and posttest questions appeared in both English and Afrikaans. Some students come from wellresourced schools which offer advanced mathematical training, and some students come from poorly resourced schools.

\section{B. Protocol and Instruments}

The entire study took place during one lecture. Students were given 12 minutes to complete a pretest. They then received a 20 minute interactive lecture on the Ratio Table, and finally wrote a 12 minute posttest. Before beginning the posttest, students were explicitly instructed to use the Ratio Table technique for solving the problems, regardless of whether they could solve the problem using an alternative technique.

The pre- and posttests both consisted of 8 multiple choice questions. Questions were categorized as dealing with numeric ratios, algebraic ratios, and/or units involving "per”. Five questions from the pretest are shown below. The categorizations, in square brackets, were not included in the original pretest. The multiple choice answer format is only shown for question 3.

1. [Numeric] There are $20 \mathrm{~kg}$ of a substance in $1 \mathrm{~m}^{3}$. How many cubic metres $\left(\mathrm{m}^{3}\right)$ does $32 \mathrm{~kg}$ of the substance occupy?

2. [Algebraic] At a party, a group of 60 students are sharing $N$ large cheese pizzas. Assuming that the students share the pizza evenly, how can you figure out the average number of students a single pizza should feed?

3. [Algebraic] During physics lab, you suspend a block from a spring and start the block moving up and down. The motion repeats itself over and over. You find that 7 cycles occur in $T$ seconds. How would you determine the number of cycles it completes in $Y$ seconds?
a. $\boldsymbol{Y} \times \boldsymbol{T} \times 7$
b. $\frac{7 \times Y}{T}$
c. $\frac{T \times 7}{Y}$
d. $\frac{Y \times T}{7}$ 
4. [Units] A student performs a motion experiment with a motorized, toy car. The car moves at a steady rate, traveling $60 \mathrm{~cm}$ in 2.4 seconds. The student divides 2.4 by 60 and gets 0.04 . Which of the following statements about the number 0.04 are true?

I. 0.04 is the distance travelled in 1 second.

II. 0.04 is the toy car's speed.

III. 0.04 is the number of seconds required for $1 \mathrm{~cm}$ of travel.

6. [Units+Algebraic] A sound speaker emits a sound wave of power $4 \mathrm{~W}$ (i. e. $4 \mathrm{~J} \mathrm{~S}^{-1}$ ) from the middle of a square room. How would you calculate how many joules of energy it will emit in $\boldsymbol{T}$ minutes?

The posttest questions were designed to be identical in structure and category, but to differ in surface content. An identical repeated measure design was not used. It was thought that confronting the exact same question in the space of one hour constituted a greater risk to validity than a difference in surface content. Posttest questions 2, 3 and 4 are shown below:

2. [Algebriac] You go to the farmer's market to buy olive oil. When you arrive you realize that you have only one dollar in your pocket. The clerk sells you 0.26 pints of olive oil for one dollar. You plan next week to buy $\boldsymbol{P}$ pints of olive oil. To figure out how much this will cost (in dollars), you should

3. [Algebraic] During physics lab, you suspend a block from a spring and start the block moving up and down. The motion repeats itself over and over. You find that $\boldsymbol{B}$ cycles occur in 10 seconds. How would you figure out the number of seconds required for a $\mathbf{C}$ cycles?

4. [Units] Your younger brother is doing his Gr. 8 homework. He is trying to calculate the density of a particular type of wood. He is told that 10 cubic metres (i.e. $10 \mathrm{~m}^{3}$ ) of this type of wood has a mass of $4 \mathrm{~kg}$. He divides the 10 by 4 to get a value of 2.5 . Which of the following statements about the number 2.5 are correct? I. 2.5 is the density of the wood II. 2.5 is the number of cubic metres that $1 \mathrm{~kg}$ of this wood will fill.

III 2.5 is the number of kilograms of this wood that will fit in $1 \mathrm{~m}^{3}$

\section{Intervention: The Ratio Table}

The most important aspect of the Ratio Table is the mapping of two physical quantities to separate columns of a table. For example, in answering question 3 of the pre-test, one would map the physical quantities of 'no. of cycles' and 'time [in seconds]' to columns, as shown in Fig. 1 below. One then enters the information provided; in this case 7 cycles ‘is to' $T$ seconds:

\begin{tabular}{|c|c|c|}
\hline \# Cycles & $:$ & Time [s] \\
\hline 7 & $:$ & $T$ \\
\hline & $:$ & \\
\hline
\end{tabular}

FIG. 1. The first step of constructing a Ratio Table.

This step is crucial and becomes very important when dealing with units for quantities such as density $\left[\mathrm{kg} \mathrm{m}^{-3}\right]$, or power $\left[J s^{-1}\right]$. Research has shown that students do not understand that $x \mathrm{~kg} \mathrm{~m}^{-3}$ can be written as $x$ kilograms per (for every) 1 cubic metre [9]. Writing units as a relationship between two physical quantities is easily accomplished using the Ratio Table. Next, students are asked to identify the quantity they require. In question 3 , they are required to calculate the number of cycles that are completed in $Y$ seconds. However, if we insert $Y$ in the right-hand column, it is not obvious how we get from $T$ seconds to $Y$ seconds. Students are therefore first introduced to a method called “Take It To One” (TITO). If we insert 1 into the right-hand column, we must apply the simple procedure of dividing by $T$ in order to get from $T$ seconds to 1 second. The golden rule of the Ratio Table is: "what we multiply/divide by on the right-hand side, we multiply/divide by on the left-hand side.” Thus, students will end up with a row showing $\frac{7}{T}$ cycles is to 1 second; see Fig. 2. Students are advised that when a row of the Ratio Table contains a "1", there is an opportunity for meaning making. In this case, $\frac{7}{T}$ cycles per 1 second represents the physical quantity of frequency. Once they have used TITO, it is easy for students to see that in order to insert $Y$ seconds into the right hand column, they need to multiply the 1 by $Y$. Following the golden rule of the Ratio Table, they finally acquire their answer: $\frac{7 \times Y}{T}$ cycles 'is to' $Y$ seconds. The interactive nature of the lecture involved posing concept tests at strategic intervals, and facilitating peer instruction [10].

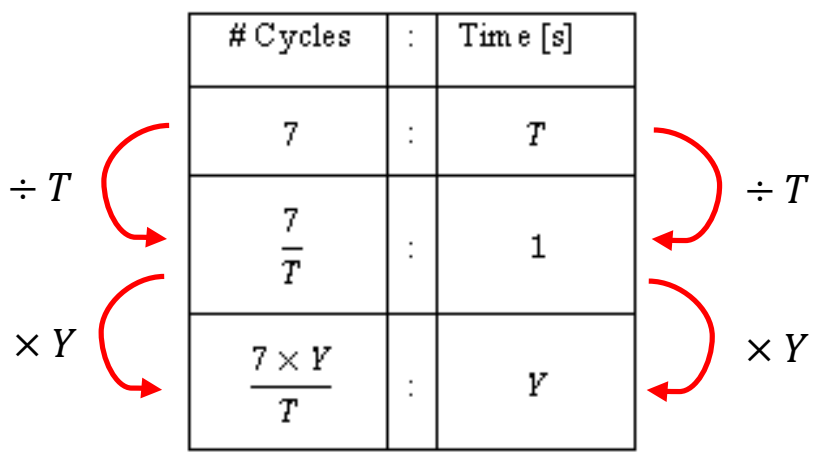

FIG. 2. The Ratio Table solution for question 3 of pre-test.

\section{RESULTS}

\section{A. Pretest results}

As observed in the pilot study, students did not struggle with the question involving numerical ratios, with $94 \%$ of 
students obtaining the correct answer for question 1 of the pre-test. However, as shown in Fig. 3 below, the results from the other questions involving algebraic ratios and/or units are significantly worse. (All error bars in all figures are $95 \%$ confidence intervals. Question number 5 is not included in the analysis due to a wording and translation error.)

The 311 students achieved an overall average of 56\% ( $\sigma=25.7)$ for the pretest. However, the histogram of marks did not produce a smooth bell-curve. The students were therefore divided into those who achieved less than $50 \%$ for the pre-test ( $n=172$, or $55 \%$ of students) and those that achieved greater than $50 \%$ for the pre-test ( $n=139$, or $45 \%$ of students). The former group achieved an overall average of $38 \%(\sigma=15.0)$ and the latter group an overall average of $80 \% \quad(\sigma=12.1)$.

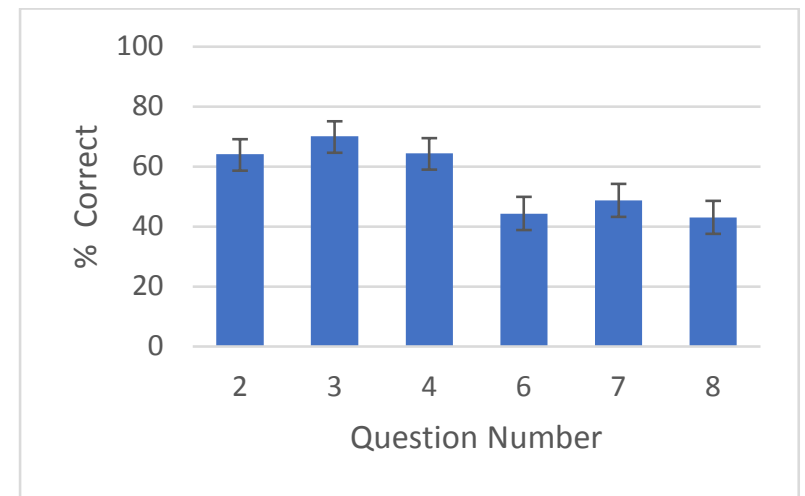

FIG. 3. Pre-test results for all students.

\section{B. Posttest results}

I have called "Group A" those 172 students who achieved less than $50 \%$ in the pretest, and "Group B" those 139 students who achieved greater than $50 \%$ in the pretest. Looking at the posttest results, Group A obtained an overall gain of 29 percentage points, achieiving an overall posttest average of $67 \%$. Group B showed a negative gain of 9 percentage points, obtaining an overall posttest average of $71 \%$. Both gains are statistically significant $(p<0.05$, paired t-test). The pre- and posttest results for individual questions are shown in Fig. 4 and Fig. 5 below.

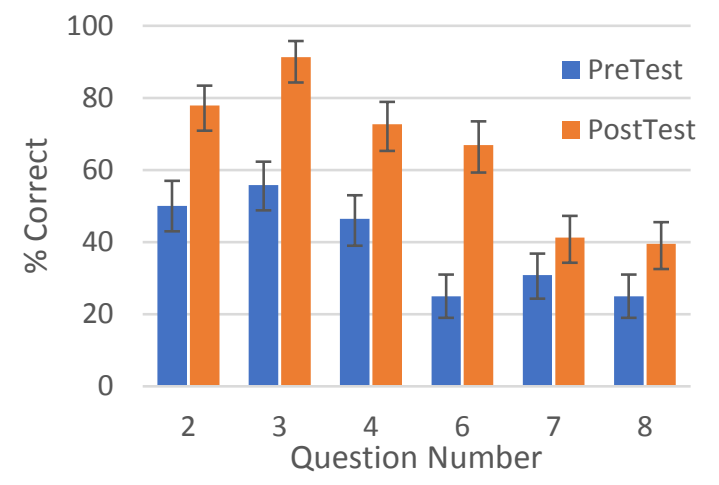

FIG. 4. Pre- and post-test results for Group A $(n=172)$

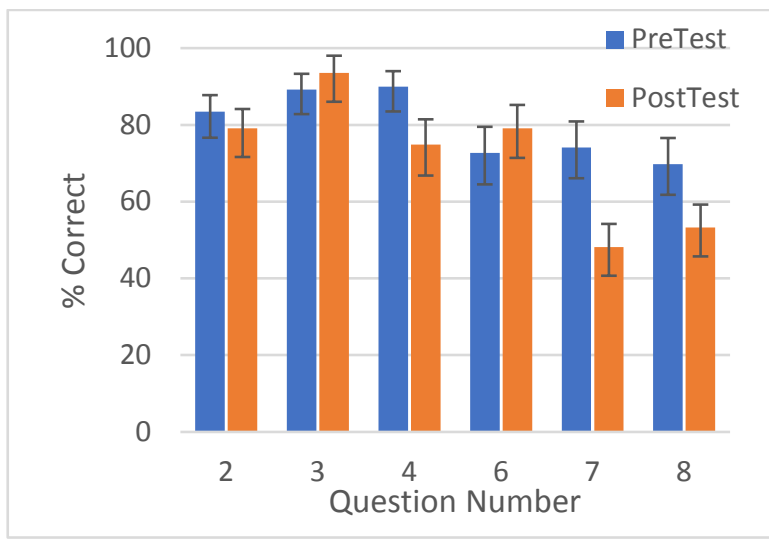

FIG. 5. Pre- and posttest results for Group B $(n=139)$

\section{DISCUSSION}

The results from question 1 involving a numerical ratio (pre-test 94\%, post-test 97\%) were not included in the final analysis as all students evidently have a productive technique for dealing with these types of questions. It is assumed they use some variation of the "cross-multiplication method'. This confirms the results from the pilot study, namely that it is helpful to draw a distinction between questions involving numerical ratios and those involving algebraic ratios. Figure 3 clearly shows that students struggle with the latter, as well as with making meaning of units involving ratios, such as seconds per meter $\left(\mathrm{s} \mathrm{m}^{-1}\right)$.

The most striking result is the marked difference between the performance of Group A and Group B, with the latter group outperforming the former by $110 \%$ in the pretest. Consider question 6 involving the units of power, Watts, in the form of an algebraic ratio, $J s^{-1}$. Group A achieved a pretest average of $25 \%$ for this question. Evidently these students do not have a technique for dealing with this type of question involving ratios. Compare this with their posttest result of $67 \%$. Group B had a pre- and posttest result of $73 \%$ and $79 \%$ respectively. This is not a statistically significant gain ( $p>0.05$, McNemar's Test). The other questions follow a similar trend: students in Group A achieve significant gains, while the pre- and posttest scores of students in Group B do not show significant differences except for questions 4, 7 and 8 (discussed below). One possible explanation for the substantial difference between Group A and Group B is the bifurcated schooling system in South Africa. Figure 6 is taken from an article by Nic Spaull titled "Education in SA: A Tale of Two Systems" [11]. (The figure is credited to Stephen Taylor [12]). It clearly indicates that South Africa's current schooling system bears the scars of a racially divided past. Ex-DET/Homeland schools constitute a dysfunctional schooling system with very poor numeracy levels, while historically white schools constitute a functioning system with high numeracy levels. The challenge for tertiary education is self-evident; important initiatives have broadened access to higher education, and 
while all freshman students may share the same potential and will to succeed, university lecturers ought to be cognizant of the fact that the students in their class have emerged from a wide variety of high school experiences, and some mathematical skills (such as proportional reasoning) might not be as consolidated as expected.

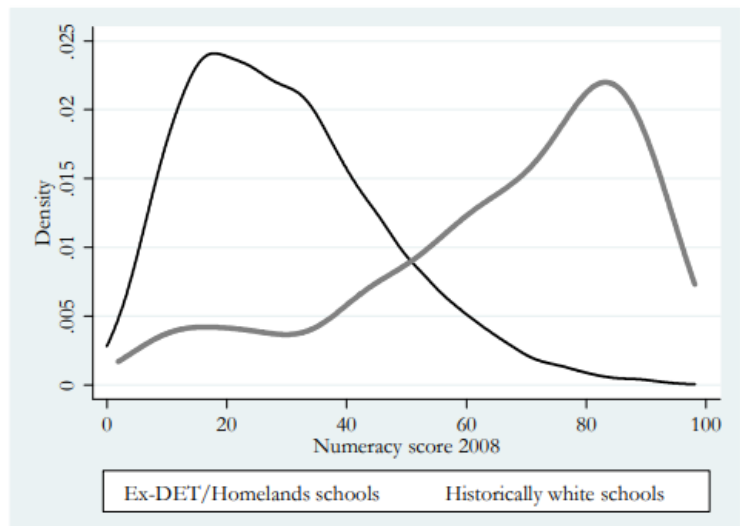

FIG. 6. Kernel density plot showing bimodal distribution of numeracy abilities of South African Grade 4 learners in 2008. Used with permission from [12].

Whatever the underlying cause of the large variation in student performance in the pretest, the benefit of the ratio table seems dependent on students' prior ability. This dependency is illustrated in Fig. 7 below, which plots percentage gain $\left[\frac{\text { posttest-pretest }}{\text { perfect posttest-pretest }}\right]$ against the number of pretest questions correctly answered. The size of the data points is proportional to the number of students represented in that data point. For 5 or more pretest questions correct, the gain was zero or negative, and therefore not included in the figure.

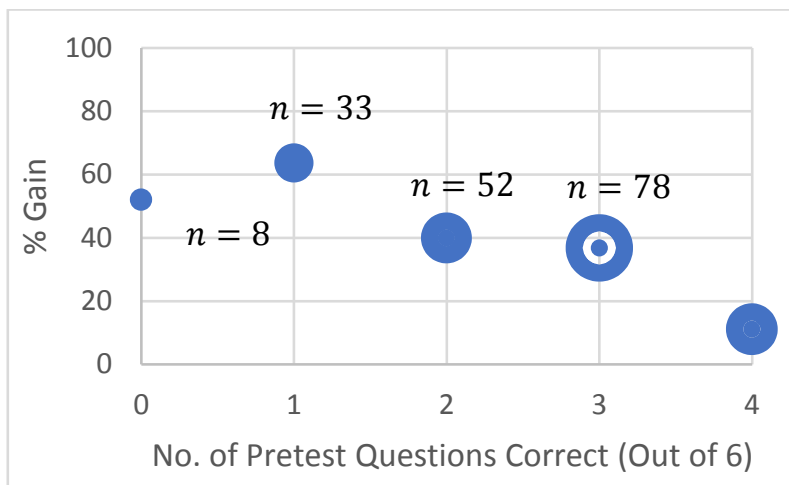

FIG. 7. Percentage gain as a function of number of correct pretest questions.

Finally there is a worrying drop in performance for Group B for questions 4, 7 and 8 (all statistically significant, $p<0.017$, McNemar's Test with Bonferroni correction). The drop in performance for questions 7 and 8 is hypothesized to be a result of time pressure. As mentioned above, students were instructed to use the Ratio Table to answer all questions in the posttest, regardless of whether they might have another, more familiar method. The time limit was strictly kept to 12 min. Note that unanswered questions (i.e. "blanks”) were scored as incorrect. Thus, the enforced use of the somewhat unfamiliar Ratio Table technique may have slowed some of the Group B students down, preventing them from completing the posttest in the allotted time. There is no obvious cause for the drop in performance for question 4. Importantly, outside of the study the Ratio Table is not intended to be a strict structure for students to adhere to, but a flexible construct for students to manipulate. It is intended to provide a foundation for proportional reasoning, if required. It is also intended to formalize a particular type of technique for dealing with ratios, in the hope that its affordances may be contrasted with those of other techniques, such as dimensional analysis.

\section{CONCLUSION}

The first conclusion from this study is that over half the freshman engineering students at Stellenbosch University have significant difficulties with basic ratios involving algebraic values. This result is important because the other half of the class does not appear to have these difficulties, which obscures the possible need for an intervention. However, as ratios are a basic threshold concept [13] it is incumbent upon teaching staff to ensure that all students have a robust technique for coping with ratios before moving on to more advanced content.

The second conclusion is that a brief intervention introducing a technique dubbed the Ratio Table can have a significantly positive impact upon students' abilities to cope with basic ratios. Importantly the intervention in this study was only 20 min long. Ideally this intervention should be introduced over a couple of lectures, and re-enforced at appropriate intervals.

The next step of this study is to formally introduce the Ratio Table in both freshman engineering physics and engineering chemistry. In a repeated measures study, an identical test will be applied at the beginning and at the end of the semester. The time limit for both pre- and posttest will be increased, which should hopefully result in positive (or at least non-negative) gains for all students.

\section{ACKNOWLEDGEMENTS}

Thank you to Dr. P. Neethling, Dr. P Chellan, Ms. M Cloete, Dr. T Grobelaar, and Ms. J van Wyk 
[1] A. B. Arons, "Student patterns of thinking and reasoning," Phys. Teach., 21, 9 (1983)

[2] A. Boudreaux, S. Kanim, and S. Brahmia, "Student facility with ratio and proportion: Mapping the reasoning space in introductory physics," arXiv Preprint (2016).

[3] F. Tourniaire and S. Pulos, "Proportional reasoning: A review of the literature,” Educ. Stud. Math., 16, 2 (1985).

[4] P. Thompson and L. Saldana, "Fractions and multiplicative reasoning," in Research Companion to the Principles and Standards of School Mathematics, edited by J. Kilpatric, G. Martin, and D. Schifter (Reston, VA, 2003).

[5] S. Brahmia, A. Boudreaux, and S. Kanim, "Obstacles to Mathematization in Introductory Physics,” arXiv Preprint (2016).

[6] M. Simon and G. Blume, "Mathematical modeling as a component of understanding ratio-as-measure: A study of prospective elementary teachers,” J. Math. Behav., 13, 2, (1994).
[7] S. Brendezel, "The relationship between proportional reasoning and visual spatial ability,” $\mathrm{PhD}$ Thesis, Rutgers (1981)

[8] E. Torigoe and G. Gladding, "Same to Us, Different to Them: Numeric Computation versus Symbolic Representation,” AIP Conference Proceedings, (2007)

[9] A. Akatugba and J. Wallace, "Mathematical dimensions of students' use of proportional reasoning in high school physics,” Sch. Sci. Math., 99, 1, (1999).

[10] E. Mazur and M. D. Somers, "Peer Instruction: A User's Manual,” Am. J. Phys., 67, 4, (1999).

[11] N. Spaull, Education in SA: A tale of two systems. 2012.

[12] S. Taylor, "Uncovering indicators of effective school management in South Africa using the National School Effectiveness Study,” Stellenbosch Econ. Work. Pap., (2011)

[13] R. Land, J. Meyer, and C. Baillie, Threshold Concepts and Transformational Learning, (Sense, Boston, 2010) 